one. The fact that doctors perceived that they listened, explained things and gave advice less, and gave other help and examined patients more when the patients were from social classes IV and V may reflect the doctors' tendency with patients from these social classes to undertake physical activity more and educate patients less. This trend, and the fact that the doctors gave explanations more to men than to women, may reflect difficulties for the four male doctors from social class $I$ in relating to patients of a different sex and different social classes.

The fact that the proportion of patients who were aware of the nature of their problem rose from $28 \%$ before the consultation to only $32 \%$ after the consultation suggests that the consultation had little educative value. When we look at the causes patients perceived for their problems, however, the most frequently mentioned causes were infection, trauma, stress and social problems, physical and environmental factors, and pregnancy. These relate to a belief that health is governed largely by external factors not under the control of the individual. ${ }^{10}$ With regard to lifestyle factors that affect health, such as obesity, alcohol, and smoking, these were perceived by doctors - but not by patients - to cause problems. Only in the case of smoking was this view adopted by patients after the consultation.

The wide divergence between doctors and patients about how ill the patient is, the cause and nature of the problem, and the content of the consultation emphasises the gap between doctors' and patients' perceptions. The doctors participating in this project clearly need to study and adjust their consultation styles to communicate better with their patients.

\footnotetext{
1 McWhinney IR. Changing models: The impact of Kuhn's theory on medicine. Fam Pract 1983;1:3-8.

Zola IK. Pathways to the doctor--from person to patient Soc Sci Med 1973:7:677-89.

Stewart MA, McWhinney IR. Buck CW. How illness presents: a study of patient behaviour. F Fam Pract 1975;2:411-4

4 Johnson SM. Snow LF, Mayhew HE. Limited patient knowledge as reproduction risk factor. F Fam Pract 1978;6:855-62.

5 Walker RD. Knowledge of symptoms suggesting malignant disease amongs general practitioner patients. I R Coll Gen Pract 1982;32:163-6. general practitioner patients. F $R$ Coll Gen Pract 1982,32:163-6. Brett AS, Mattieu AE. Perceptions and behaviour of patients with upper respiratory tract infections. f Fam Pract 1982;15:277-9.

7 Johnson SS. Health beliefs of hypertensive patients in a family medicin residency programme. F Fam Pract 1979;9:877-83.

8 Al Jumail S, Martin E. Primary care in a military community in Saudi Arabia. Update 1983;27:959-64

9 Belson W, Duncan JA. A comparison of the check list and open response questioning systems. Applied Statistics 1962;11:120-32.

10 Coulter A. Lifestyles and social class: implications for primary care. $\mathcal{F} R \mathrm{Col}$ Gen Pract 1987;37:533-6.

(Accepted 23 April 1991)
}

Needs Assessment Unit in the Departments of Public Health, City and Hackney

Health Authority, and General Practice and Primary Care, The Medical Colleges of St Bartholomew's and The London Hospitals, London Ann Bowling, PHD, senior lecturer

Bobbie Jacobson, FFPH, director of public health Lesley Southgate, PHD, senior lecturer

Juliet Formby, DCR, research assistant

\section{Correspondence to:} Dr A Bowling, Department of General Practice and Primary Care, St Bartholomew's Hospital Medical College, London EC1A 7BE.

\title{
General practitioners' views on quality specifications for "outpatient referrals and care contracts"
}

\author{
Ann Bowling, Bobbie Jacobson, Lesley Southgate, Juliet Formby
}

Abstract

Objective-To ascertain general practitioners' views about which quality specifications should be included in contracts for hospital care.

Design-In depth interview study and postal survey.

Setting-General practitioners in City and Hackney Health District.

Subjects-Fourteen doctors were interviewed in depth; 77 of 131 doctors (59\%) returned postal questionnaires.

Main outcome measure-Rating of listed quality specifications.

Results-The most popular items which doctors thought should be included in contracts by April 1991 related to the availability of patients' notes in outpatient clinics, respect shown to general practitioners in telephone communications with hospital doctors, supply of medicines after discharge, patient management plans for general practitioners, the earlier arrival of discharge slips, the type of hospital doctor to see new outpatients, and the unnecessary duplication of investigations.

Conclusions-A high premium was attached by general practitioners to effective organisation, effective communication between primary and secondary sources of care, and effective communication with patients.

\section{Introduction}

The white paper Working for Patients states that in future each district health authority will be responsible for buying "the best service it can from its own hospitals, from other authorities' hospitals, from self governing hospitals, or from the private sector." Hospitals and community health services will have to satisfy districts that they are delivering a high quality and cost effective service, and their performance will be monitored by district health authorities. ${ }^{12}$ Hopkins and Maxwell have given examples of measures that patients might like to see specified in a contract for service (for example, length of waiting lists, quality of explanations given, standards of care). ${ }^{3}$ They suggested that the main considerations of quality in drawing up contracts for services should include access to services, communication, technical safety and effectiveness, hotel services, discharge procedures, aftercare, patien outcome, equity, relevance to need, and value for money.

General practitioners are the gatekeepers of the National Health Service. Most patients who attend hospital outpatient departments and many emergency and most non-emergency inpatient hospital admissions of patients are referred by general practitioners. This, along with the proposal for general practitioners to hold their own budgets, has major implications for use of resources. The National Health Service and Community Care Act 1990 acknowledges the importan part played by the general practitioner in acting as proxy for the health service user, and health authorities are expected to consult local general practitioners over the development and content of contracts for health care. $^{4}$

\section{Present study}

Members of the general practitioners' forum and the purchasing group in the City and Hackney Health District commissioned the survey of general practitioners presented here. The aim was to ascertain general practitioners' views about which quality specifications should be included in contracts for hospital care. An underlying aim was to assess how much information, and of what quality, could be obtained in the time scale imposed by the need to set contracts for April 1991. This led to a combined approach of in depth interviews and a postal survey. 


\section{Methods}

A pilot study was carried out based on in depth interviews with 14 general practitioners in the district who represented a wide geographical spread. They agreed to be interviewed after the study was discussed at a meeting between local doctors, the Department of Public Health, and the purchasing group of the district health authority on purchasing health care. The study was welcomed as a joint initiative on the part of the general practice and the purchasing group.

The pilot study took place over three weeks in the spring of 1990. General practitioners were asked open questions about what standards of service they expected when they referred patients to hospital outpatient departments and why they referred patients to hospitals and departments. These issues were probed in depth, and doctors were encouraged to provide anecdotes about "good" and "bad" referrals. They were assured that all information would be treated with the strictest confidence. The criteria of quality thus elicited then formed the basis of a list for inclusion in a postal questionnaire, which was sent to all general practitioners in the district.

In June 1990 postal questionnaires were sent to all 131 general practitioners within City and Hackney Health District. After three weeks 46 questionnaires had been returned $(35 \%)$. Reminder questionnaires were then dispatched, and three weeks later a further 23 had been returned (18\%). Another reminder was sent but this resulted in only one more questionnaire being returned. Telephone calls were made to the remaining non-respondents, which increased the response rate by a further seven doctors $(4 \%)$. The overall response rate was $59 \%$ (77) of the 131 general practitioners, representing $66 \%$ of all practices in City and Hackney Health District.

Using non-confidential information from the (then) local family practitioner committee and the Medical
Directory, we compared respondents and nonrespondents with respect to sex, year of qualification, number of partners, and postgraduate qualifications. There were no significant differences between the two groups.

\section{Results}

The most popular items which the 77 respondents thought should be included in the contracts by April 1991 related to the percentage of patients' notes which should be available in outpatient departments, appropriate respect to be shown to general practitioners in telephone communications with hospital doctors, supply of inpatients' medicines after discharge, the inclusion of patient management plans in clinic letters to general practitioners, the earlier arrival of discharge slips, the type of hospital doctor to see new patients, and the unnecessary duplication of investigations (table). Items given the lowest priority related to the cancellation of outpatient appointments, between consultant referrals without reference to the general practitioner, and the availability of interpreters for non-English speaking patients.

In order to assess the distribution of replies by practice one responding doctor per practice was randomly sampled to represent that practice (in the case of practices with more than one responding doctor). The table gives the distribution of replies by practice. These were similar to those for all doctors

Although the general philosophy underlying the inclusion of criteria of quality in the new contracts was developed by the purchasing group early on, the specific items eventually included in the contracts reflected the ongoing dialogue between the general practitioners' forum and the purchasing group using the data presented here.

City and Hackney Health Authority's draft document for consultation "Contracts for purchasing health care

Results of survey of general practitioners' views on quality specification for outpatient referrals and care contracts, City and Hackney

\% Of general practitioners indicating that specification should be
mandatory by:

Discharge slips to be received within one week of all patients leaving hospital. Information on diagnosis and drugs prescribed to be included

Appropriate respect to be shown to general practitioners in telephone communications

All inpatients being discharged should be given two weeks' supply of current medicines

All new patients to be seen by consultant or registrar

Over $90 \%$ of patients should be seen in outpatients with their notes available

Clinic letters to general practitioners must include a management plan for each patient and reply to any questions raised by general practitioners in the referral letter

Outpatients needing immediate treatment must be given at least two weeks' supply of drugs before leaving clinic

No investigations (for example, by general practitioner) should be unnecessarily duplicated by hospital doctors

A list of each department's available services to be sent to general practitioners

Protocols to be designed and made available for general practitioners on how to obtain urgent access to outpatients

Informed consent to be obtained for all procedures (to be assessed by consumer surveys)

Notice of at least one week to be given for patients having elective treatment/surgery

Appropriate communication with patients by all staff (to be assessed by consumer surveys)

Patients must be referred back to their general practitioners as soon as appropriate (to be monitored)

No patient attending outpatients to see doctor should be seen or followed up solely by research nurse

Appropriate respect to be shown for patients' autonomy by all staff (to be assessed by consumer

surveys)

General practitioners to be sent monthly updates on waiting time

No patients to arrive for booked admission and sent home without treatment/surgery (for example, due to bed cancellation

Continuity of medical care in outpatients should be carefully monitored

Patients referred to named consultants should be seen by those consultants

Interpreters/information in all languages spoken locally to be available

$80 \%$ Of patients to be seen within half an hour of appointment time

Between consultant referrals should not be made without consulting the general practitioner when appropriate

Patients' outpatient appointments must not be cancelled and remade without informing general

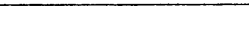

2

\begin{tabular}{rrrrrrrr}
96 & 89 & 2 & 3 & - & - & 2 & 8 \\
87 & 86 & 2 & 3 & - & - & 11 & 11 \\
87 & 81 & 5 & 8 & - & - & 8 & 11 \\
86 & 81 & 10 & 11 & - & - & 4 & 8 \\
85 & 81 & 12 & 14 & - & - & 3 & 5 \\
84 & 78 & 11 & 16 & 1 & - & 4 & 5 \\
81 & 81 & 9 & 8 & - & - & 9 & 11 \\
80 & 73 & 9 & 11 & 2 & 2 & 9 & 14 \\
76 & 72 & 13 & 14 & 3 & - & 8 & 14 \\
75 & 73 & 17 & 16 & - & - & 8 & 11 \\
74 & 61 & 16 & 19 & 3 & 6 & 7 & 14 \\
69 & 64 & 20 & 25 & 4 & 3 & 7 & 8 \\
69 & 64 & 14 & 17 & 4 & 2 & 13 & 17 \\
67 & 67 & 21 & 11 & - & - & 12 & 22 \\
66 & 72 & 13 & 14 & - & - & 21 & 14 \\
65 & 64 & 24 & 25 & 4 & 3 & 7 & 8 \\
64 & 73 & 17 & 8 & 3 & 3 & 16 & 16 \\
64 & 72 & 26 & 14 & 6 & 6 & 4 & 8 \\
64 & 59 & 31 & 35 & 1 & - & 4 & 6 \\
57 & 59 & 28 & 26 & 1 & 3 & 14 & 12 \\
53 & 44 & 32 & 39 & 3 & 3 & 12 & 14 \\
50 & 55 & 42 & 28 & 1 & 3 & 7 & 14 \\
49 & 47 & 17 & 11 & - & - & 34 & 42 \\
49 & 49 & 24 & 19 & 1 & - & 35 & 32 \\
\hline
\end{tabular}

Numbers of respondents to each item varied from 71 to 77 general practitioners and to 35 to 38 practices. 
- $90 \%$ of patients should normally be seen within half an hour of their appoint ment times

- No patient should be seen by a senior house officer unsupervised either at the first or follow up appointments and the substantive response to the referring general practitioner should come from a more senior clinician

- Medical records for individual patients complete with relevant reports should be available on arrival on $90 \%$ of occasions

- An explicit commitment should be made to giving patients a clear explanation of their condition and treatment(s) on offer

- Each clinic or group of clinics should develop a strategy for ensuring that patients are treated with courtesy by all
Prioritised by general practitioners

$80 \%$ Of patients to be seen within half an hour of appointment time (50\%)

All new patients to be seen by consultant or registrar $(86 \%)$

Over $90 \%$ of patients should be seen in outpatients with their notes available $(85 \%)$

Appropriate communication with patients by all staff (to be assessed by consumer surveys) $(69 \%)$

and

Informed consent to be obtained for all procedures (to be assessed by con sumer surveys) $(74 \%)$

Appropriate respect to be shown for patients' autonomy by all staff (to be assessed by consumer surveys) (65\%)

Overlap between quality criteria for inclusion in contracts published in district health authority's draft document on purchasing and quality criteria prioritised by general practitioners. (Percentages of doctors who thought item mandatory for inclusion by A pril 1991 given in parentheses) services," issued in November 1990, contains a "general philosophy of care" which takes into account several of the criteria which were popular among the general practitioners - for example, "Patients should be treated with courtesy and sensitivity "; "A patient's time should be valued at least as much as staff time"; "Services provided should be efficient and convenient"; "Staff and services should be sensitive to the needs of people of different cultural/ethnic backgrounds." The figure shows the urgent requirements for inclusion in the contracts which are listed in the document and which reflect criteria specified by general practitioners.

Eighty per cent or more of general practitioners prioritised the items relating to the supply of medicines on discharge, the provision of management plans, the timing of discharge slips, elimination of unnecessary duplication of investigations, and appropriate respect to be shown to general practitioners in telephone communications. Discussions about including these items in future contracts are still taking place. Discussions about responsibility for prescribing and supply of medicines on discharge from hospital are proving the most difficult.

\section{Discussion}

This paper presents data on general practitioners' views on criteria of quality to be included in the new contracts for outpatient care. Different methodological approaches were adopted in order to obtain and combine potentially useful information within a restricted time period. The response to the postal survey was $59 \%$ of doctors, or $66 \%$ of all practices in the district. Although less than ideal, this was regarded as a good response. General practitioners are reluctant to complete questionnaires, partly because of the volume that they routinely receive.

It is difficult to see how the response rate among the general practitioners could have been improved. Nonrespondents were sent two reminder letters and additional copies of the questionnaire, and they also received a telephone call. Possibly a shorter questionnaire might have increased the response slightly. The questionnaire asked for details of referral preferences and practices for selected medical conditions as well as for the doctors' views on criteria for the contracts (the aim was to provide additional information which would facilitate the negotiation of contracts and to supplement data routinely available on referral patterns for inpatient episodes). Thus the penalty for combining two related topics into one questionnaire may be a lower response rate.

The doctors' replies to the items on criteria for quality indicate that high premium is attached to efficient organisation (for example, the availability of notes in outpatient departments, supply of medicines, management plans, and the arrival of discharge slips), effective communication between primary and secondary sources of care, and the ways in which people are treated in hospitals.

Most doctors wanted most of the listed standards to be included in the new contracts by April 1991 and most of the remainder to be included by April 1993. These results led to specifications for setting contracts and discussions between purchasers and providers. It is essential that these criteria are taken seriously or they will lead to the alienation of general practitioners. If note is taken of these results then this can lead to increased efficiency and humanity of the longer term management plan. Short term expediency-that is, avoiding dealing with these issues-leads to longer term wastage of resources. As Klein has stated, "It is the purchasing agreements and contracts that should answer the question of what is being provided to whom and provide information about changes in levels of service, access, availability, and standards over time."'

The pilot study of doctors who were interviewed in depth indicated that their current referral practices reflected convenience of access for the patient (that is, nearby hospitals) as well as the quality of the service provided-for example, if a local surgeon was known to be rude to patients, then patients (particularly the more sensitive ones) were said to be referred elsewhere. Unpublished analyses of the general practitioners' stated preferred places of referral and their actual referral patterns showed that they largely referred to the hospitals and departments of their choice. It will be interesting to review the situation under the new system and monitor whether referral patterns change when general practitioners are more able to incorporate quality specifications in their referral criteria as hospitals start responding to the contracting process.

Relationships between members of the purchasing group and most of the general practitioners within the district of the study were generally good despite the difficulties of working in an area of high deprivation. Collecting information vital for planning and negotiating contracts with agreed criteria are likely to be much more difficult in districts where these conditions are not met. What will be the effect the moment a general practitioner cannot refer to his or her and the patient's chosen hospital, especially if this represents a change in place of treatment? The consequence may be that the general practitioner will see fundholding as the only solution. This may seem desirable to some, but the public health and needs based approach which ensures that contracts reflect priorities for health on a wider basis than just one general practice will be lost. Fundholding practices will have a responsibility to remember public health priorities and act as advocates for other practices in improving the quality of services, and not merely act as competitors.

\footnotetext{
Department of Health. Funding and contracts for hospital services. Workin paper 2. London: HMSO, 1989

2 Secretaries of State for Health, Wales, Northern Ireland, and Scotland. Working for patients. London: HMSO, 1989. (Cm 555.)

3 Hopkins A, Maxwell R. Contracts and quality of care BMF 1990;300:919-22.

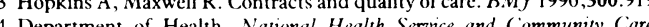

5 Klein R. The politics of change. $B M \gamma$ 1991;302:1102-3.

Accepted 24 Mav 1991
} 